\title{
Hedonic Prices and Implicit Markets: Product Differentiation in Pure Competition
}

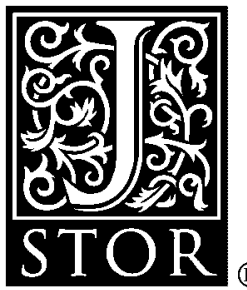

\section{Sherwin Rosen}

The Journal of Political Economy, Vol. 82, No. 1. (Jan. - Feb., 1974), pp. 34-55.

Stable URL:

http://links.jstor.org/sici?sici=0022-3808\%28197401\%2F02\%2982\%3A1\%3C34\%3AHPAIMP\%3E2.0.CO\%3B2-U

The Journal of Political Economy is currently published by The University of Chicago Press.

Your use of the JSTOR archive indicates your acceptance of JSTOR's Terms and Conditions of Use, available at

http://www.jstor.org/about/terms.html. JSTOR's Terms and Conditions of Use provides, in part, that unless you have obtained prior permission, you may not download an entire issue of a journal or multiple copies of articles, and you may use content in the JSTOR archive only for your personal, non-commercial use.

Please contact the publisher regarding any further use of this work. Publisher contact information may be obtained at http://www.jstor.org/journals/ucpress.html.

Each copy of any part of a JSTOR transmission must contain the same copyright notice that appears on the screen or printed page of such transmission.

The JSTOR Archive is a trusted digital repository providing for long-term preservation and access to leading academic journals and scholarly literature from around the world. The Archive is supported by libraries, scholarly societies, publishers, and foundations. It is an initiative of JSTOR, a not-for-profit organization with a mission to help the scholarly community take advantage of advances in technology. For more information regarding JSTOR, please contact support@ jstor.org. 


\title{
Hedonic Prices and Implicit Markets: Product Differentiation in Pure Competition
}

\section{Sherwin Rosen}

\author{
University of Rochester and Harvard University
}

\begin{abstract}
A class of differentiated products is completely described by a vector of objectively measured characteristics. Observed product prices and the specific amounts of characteristics associated with each good define a set of implicit or "hedonic" prices. A theory of hedonic prices is formulated as a problem in the economics of spatial equilibrium in which the entire set of implicit prices guides both consumer and producer locational decisions in characteristics space. Buyer and seller choices, as well as the meaning and nature of market equilibrium, are analyzed. Empirical implications for hedonic price regressions and index number construction are pointed out.
\end{abstract}

\section{Introduction and Summary}

This paper sketches a model of product differentiation based on the hedonic hypothesis that goods are valued for their utility-bearing attributes or characteristics. Hedonic prices are defined as the implicit prices of attributes and are revealed to economic agents from observed prices of differentiated products and the specific amounts of characteristics associated with them. They constitute the empirical magnitudes explained by the model. Econometrically, implicit prices are estimated by the firststep regression analysis (product price regressed on characteristics) in the construction of hedonic price indexes. With few exceptions, structural

The substance of this paper arose from conversations with $\mathrm{H}$. Gregg Lewis several years ago. A multitude of other people have contributed advice and criticism. Among them are William Brock, Stanley Engerman, Robert J. Gordon, Zvi Griliches, Robert E. Lucas, Jr., Michael Mussa, and the referee. Remaining errors are my own responsibility. Financial support from the Center for Naval Analysis and the National Institute of Education is gratefully acknowledged. 
interpretations of the hedonic method are not available. ${ }^{1}$ Therefore, our primary goal is to exhibit a generating mechanism for the observations in the competitive case and to use that structure to clarify the meaning and interpretation of estimated implicit prices. It will be shown that these data generally contain less information than is commonly supposed. However, the model suggests a method that often can identify the underlying structural parameters of interest. Also, as a general methodological point, it is demonstrated that conceptualizing the problem of product differentiation in terms of a few underlying characteristics instead of a large number of closely related generic goods leads to an analysis having much in common with the economics of spatial equilibrium and the theory of equalizing differences.

The model itself amounts to a description of competitive equilibrium in a plane of several dimensions on which both buyers and sellers locate. The class of goods under consideration is described by $n$ objectively measured characteristics. Thus, any location on the plane, is represented by a vector of coordinates $z=\left(z_{1}, z_{2}, \ldots, z_{n}\right)$, with $z_{i}$ measuring the amount of the $i$ th characteristic contained in each good. Products in the class are completely described by numerical values of $z$ and offer buyers distinct packages of characteristics. Furthermore, existence of product differentiation implies that a wide variety of alternative packages are available. Hence, transactions in products are equivalent to tied sales when thought of as bundles of characteristics, suggesting applicability of the principle of equal advantage for analyzing market equilibrium.

In particular, a price $p(z)=p\left(z_{1}, z_{2}, \ldots, z_{n}\right)$ is defined at each point on the plane and guides both consumer and producer locational choices regarding packages of characteristics bought and sold. Competition prevails because single agents add zero weight to the market and treat prices $p(z)$ as parametric to their decisions. In fact the function $p(z)$ is identical with the set of hedonic prices- "equalizing differences"-as defined above, and is determined by some market clearing conditions: Amounts of commodities offered by sellers at every point on the plane must equal amounts demanded by consumers choosing to locate there. Both consumers and producers base their locational and quantity decisions on maximizing behavior, and equilibrium prices are determined so that buyers and sellers are perfectly matched. No individual can improve his position, and all optimum choices are feasible. As usual, market clearing prices, $p(z)$, fundamentally are determined by the distributions of consumer tastes and producer costs. We show how it is possible to recover,

${ }^{1}$ Excellent summaries of the hedonic technique are available in Griliches (1971, chap. 1) and Gordon (1973). Major exceptions to the statement in the text are those studies dealing with depreciation and obsolescence (see Griliches 1971, chaps. 7 and 8) and some recent models based on markup pricing (e.g., Ohta and Griliches 1972). 
or identify, some of the parameters of these underlying distributions by a suitable transformation of the observations.

An early contribution to the problem of quality variation and the theory of consumer behavior has been made by Houthakker (1952). His analysis is designed to take account of the fact that consumers purchase truly negligible fractions of all goods available to them without having to deal with a myriad of corner solutions required by conventional theory. That virtue of Houthakker's treatment is preserved in the present model. More recently Becker (1965), Lancaster (1966), and Muth (1966) have extended Houthakker's methods to more explicit consideration of utilitybearing characteristics. Again, the emphasis is on consumer behavior and properties of market equilibrium have not been worked out, a gap we hope to fill, in part, here. The spirit of these recent contributions is that consumers are also producers. Goods do not possess final consumption attributes but rather are purchased as inputs into self-production functions for ultimate characteristics. Consumers act as their own "middlemen," so to speak. In contrast, the model presented below interposes a market between buyers and sellers. Producers themselves tailor their goods to embody final characteristics desired by customers and receive returns for serving economic functions as intermediaries. These returns arise from economies of specialized production achieved by specialization and division of labor through market transactions not available outside organized markets with self-production.

Section II discusses individual choices in the market and the nature of market equilibrium. Some simple examples of analytic solutions for general equilibrium are given in Section III. Section IV presents an empirical method for identifying the underlying structure from the observations, while Section $\mathrm{V}$ applies the model to price index number construction in the presence of legislated restrictions. To highlight essential features, the simplest possible specifications are chosen throughout. As a further appeal to intuition, use is made of geometrical constructions wherever possible.

\section{Market Equilibrium}

Consider markets for a class of commodities that are described by $n$ attributes or characteristics, $z=\left(z_{1}, z_{2}, \ldots, z_{n}\right)$. The components of $z$ are objectively measured in the sense that all consumers' perceptions or readings of the amount of characteristics embodied in each good are identical, though of course consumers may differ in their subjective valuations of alternative packages. The terms "product," "model," "brand," and "design" are used interchangeably to designate commodities of given quality or specification. It is assumed that a sufficiently large number of differentiated products are available so that choice among various com- 
binations of $z$ is continuous for all practical purposes. That is, there is a "spectrum of products" among which choices can be made. As will be apparent, this assumption represents an enormous simplification of the problem. It is obviously better approximated in some markets than others, and there is no need to belabor its realism. ${ }^{2}$ To avoid complications of capital theory, possibilities for resale of used items in secondhand markets are ignored, either by assuming that secondhand markets do not exist, or alternatively, that goods represent pure consumption.

Each product has a quoted market price and is also associated with a fixed value of the vector $z$, so that products markets implicitly reveal a function $p(z)=p\left(z_{1}, \ldots, z_{n}\right)$ relating prices and characteristics. This function is the buyer's (and seller's) equivalent of a hedonic price regression, obtained from shopping around and comparing prices of brands with different characteristics. It gives the minimum price of any package of characteristics. If two brands offer the same bundle, but sell for different prices, consumers only consider the less expensive one, and the identity of sellers is irrelevant to their purchase decisions. Adopt the convention of measuring each $z_{i}$ so that they all may be treated as "goods" (i.e., so that consumers place positive rather than negative marginal valuations on them) in the neighborhood of their minimum technically feasible amounts. Then firms can alter their products and increase $z$ only by use of additional resources, and $p\left(z_{1}, \ldots, z_{n}\right)$ must be increasing in all its arguments. Assume $p(z)$ possesses continuous second derivatives. Since a major goal of the analysis is to present a picture of how $p(z)$ is determined, it is inappropriate to place too many restrictions on it at the outset. However, note that there is no reason for it to be linear as is typically the case. The reason is that the differentiated products are sold in separate, though of course highly interrelated, markets. This point is spelled out in some detail below.

A buyer can force $p(z)$ to be linear if certain types of arbitrage activities are allowed. Let $z_{a}, z_{b}$, and $z_{c}$ be particular values of the vector $z$. (i) Suppose $z_{a}=(1 / t) z_{b}$, and $p\left(z_{a}\right)<(1 / t) p\left(z_{b}\right)$, where $t$ is a scalar and $t>1$. Then $t$ units of a model offering $z_{a}$ yield the same amount of characteristics as a model offering $z_{b}$, but at less cost, ruling out transactions in convex portions of $p(z)$. (ii) Suppose $z_{a}<z_{b}<z_{c}$ and $p\left(z_{b}\right)>$ $\delta p\left(z_{a}\right)+(1-\delta) p\left(z_{c}\right)$, where $0<\delta<1$ and $z_{b}$ is defined by $z_{b}=$ $\delta z_{a}+(1-\delta) z_{c}$. Then characteristics in amount of $z_{b}$ could be achieved by purchasing $\delta$ units of a model containing $z_{a}$ and $(1-\delta)$ units of a model containing $z_{c}$ at lower cost than by direct purchase of a brand containing $z_{b}$, and products in concave portions of $p(z)$ would be uneconomical. Arbitrage is assumed impossible in what follows (at this point

2 This assumption was first employed by L. M. Court (1941) and allows the use of marginal analysis rather than the programming methods required by Lancaster's (1966) formulation. Following the general rule, it is not without its costs, however (see below). 
we depart from Lancaster [1966]) on the assumption of indivisibility. This amounts to an assumption that packages cannot be untied. For example, in terms of one characteristic, two 6-foot cars are not equivalent to one 12 feet in length, since they cannot be driven simultaneously (case [i]); while a 12-foot car for half a year and a 6-foot car for the other half is not the same as 9 feet all year round (case [ii]). Similarly, assume sellers cannot repackage existing products in this manner or do not find it economical to do so, as might not be the case with perfect rental markets and zero transactions and reassembly costs.

\section{A. The Consumption Decision}

To begin, suppose consumers purchase only one unit of a brand with a particular value of $z$. Write the utility function as $U\left(x, z_{1}, z_{2}, \ldots, z_{n}\right)$ assumed strictly concave, in addition to the other usual properties, where $x$ is all other goods consumed. It would not be difficult to treat $z$ as intermediate goods and relate them to yet more ultimate commodities through self-production functions, but that complication is ignored. Set the price of $x$ equal to unity and measure income, $y$, in terms of units of $x: y=$ $x+p(z)$. Maximization of utility subject to the nonlinear budget constraint requires choosing $x$ and $\left(z_{1}, \ldots, z_{n}\right)$ to satisfy the budget and the first-order conditions $\partial p / \partial z_{1}=p_{i}=U_{z_{i}} / U_{x}, i=1, \ldots, n$. Optimality is achieved by purchasing a brand offering the desired combination of characteristics. Second-order conditions are fulfilled on the usual assumptions regarding $U$, so long as $p(z)$ is not sufficiently concave (for a general statement of these conditions under a nonlinear constraint see Intriligator [1971]).

To stress the essential spatial context of the problem, define a value or bid function $\theta\left(z_{1}, \ldots, z_{n} ; u, y\right)$ according to

$$
U\left(y-\theta, z_{1}, \ldots, z_{n}\right)=u \text {. }
$$

The expenditure a consumer is willing to pay for alternative values of $\left(z_{1}, \ldots, z_{n}\right)$ at a given utility index and income is represented by $\theta(z ; u, y)$. It defines a family of indifference surfaces relating the $z_{i}$ with "money" (i.e., with $x$ foregone), and has been widely used in urban economics (e.g., see Alonso 1964). Differentiate (1) to obtain

$$
\begin{aligned}
\theta_{z_{i}} & =U_{z_{i}} / U_{x}>0, \theta_{u}=-1 / U_{x}<0, \text { and } \theta_{y}=1, \\
\theta_{z_{i} z_{i}} & =\left(U_{x}^{2} U_{z_{i} z_{i}}-2 U_{x} U_{z_{i}} U_{x z_{i}}+U_{z_{i}}^{2} U_{x x}\right) / U_{x}^{3}<0,
\end{aligned}
$$

where the inequality in (3) follows from the assumptions about the bordered Hessian matrix of $U$. Also, strict concavity of $U$ implies that $\theta$ is concave in $z$. Equations (2) and (3) show that the value function is increasing in $z_{i}$ at a decreasing rate. Alternatively, $\theta_{z_{i}}$ is the marginal rate of substitution between $z_{i}$ and money, or the implicit marginal 


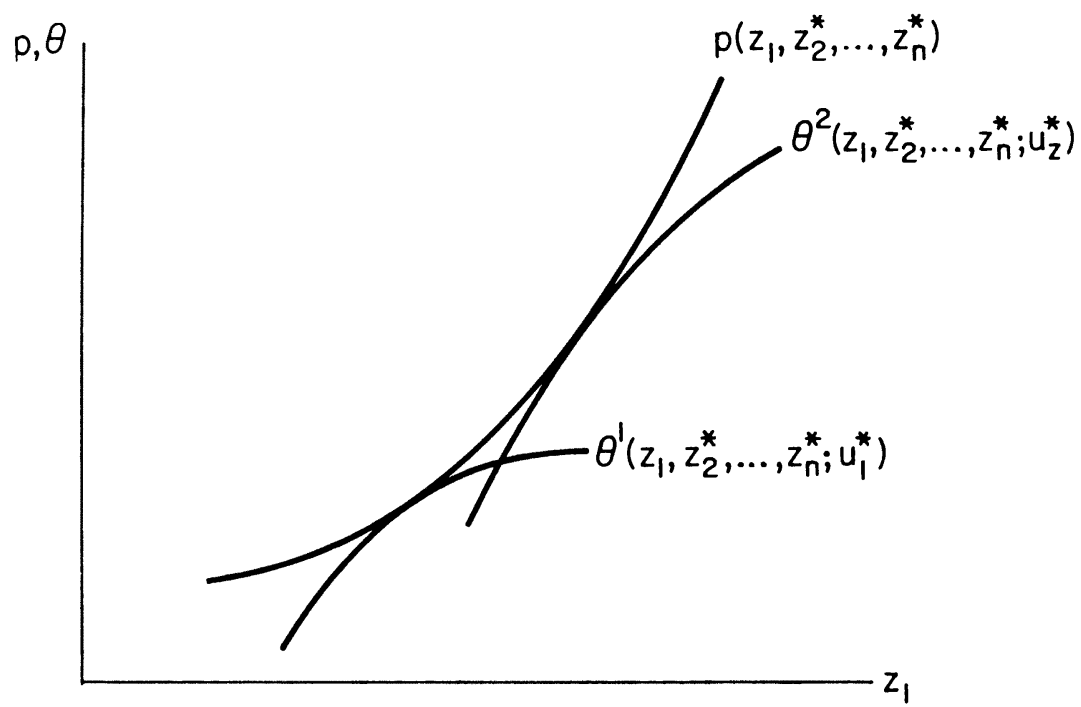

FIG. 1

valuation the consumer places on $z_{i}$ at a given utility index and income. It indicates his reservation demand price for an additional unit of $z_{i}$, which is decreasing in $z_{i}$.

The amount the consumer is willing to pay for $z$ at a fixed utility index and income is $\theta(z ; u, y)$, while $p(z)$ is the minimum price he must pay in the market. Therefore, utility is maximized when $\theta\left(z^{*} ; u^{*}, y\right)=p\left(z^{*}\right)$ and $\theta_{z_{i}}\left(z^{*} ; u^{*}, y\right)=p_{i}\left(z^{*}\right), i=1, \ldots, n$, where $z^{*}$ and $u^{*}$ are optimum quantities. In other words, optimum location on the $z$-plane occurs where the two surfaces $p(z)$ and $\theta\left(z ; u^{*}, y\right)$ are tangent to each other. One dimension of consumer equilibrium is illustrated in figure 1 , where the surfaces have been projected onto the $\theta-z_{1}$ plane cut at $\left(z_{2}^{*}, \ldots, z_{n}^{*}\right)$. A family of indifference curves, of which only one member (at $\left.u^{*}\right)$ is shown, is defined by $\theta\left(z_{1}, z_{2}^{*}, \ldots, z_{n}^{*} ; u, y\right)$. Two different buyers are shown in the figure, one with value function $\theta^{1}$ and the other with $\theta^{2}$. The latter purchases a brand offering more $z_{1} \cdot{ }^{3}$

In general, far less can be said than in the standard analysis about comparative statics, because the budget constraint is nonlinear. Differentiate $\theta_{z_{i}}$ with respect to $u, \theta_{z_{i} u}=\left(U_{x} U_{x z_{i}}-U_{z_{i}} U_{x x}\right) / U_{x}^{2}$, the numerator of which is recognized as determining the sign of the income elasticity of demand for "good" $z_{i}$ in standard theory when the other components of $z$ are "held constant." If all these derivatives are positive $\left(z_{i}\right.$ is "normal" in this restricted sense for all $i$ ), the gradient of $\theta$ unambiguously

${ }^{3}$ Lewis (1969) employs a similar construction in analyzing the problem of hours of work as a tied sale. Jobs offer a fixed wage-hour package, which varies from job to job. The market establishes a function relating wages and hours on which both workers and employers base their decisions. 
increases as $u$ increases. Additional income always increases maximum attainable utility. Hence if $p(z)$ is convex and sufficiently regular everywhere, we might expect higher income consumers to purchase greater amounts of all characteristics. Only in that case would it be true that larger income leads to an unambiguous increase in the overall "quality" consumed, and differentiated products' markets would tend to be stratified by income. However, in general there is no compelling reason why overall quality should always increase with income. Some components may increase and others decrease (cf. Lipsey and Rosenbluth 1971). Be that as it may, a clear consequence of the model is that there are natural tendencies toward market segmentation, in the sense that consumers with similar value functions purchase products with similar specifications. This is a well-known result of spatial equilibrium models. In fact, the above specification is very similar in spirit to Tiebout's (1956) analysis of the implicit market for neighborhoods, local public goods being the "characteristics" in this case. He obtained the result that neighborhoods tend to be segmented by distinct income and taste groups (also, see Ellickson 1971). That result holds true for other differentiated products too.

Allowing a parameterization of tastes across consumers, the utility function may be written $U\left(x_{1}, z_{1}, \ldots, z_{n} ; \alpha\right)$, where $\alpha$ is a parameter that differs from person to person. Equilibrium value functions depend on both $y$ and $\alpha$. A joint distribution function $F(y, \alpha)$ is given in the population at large, and equilibrium of all consumers is characterized by a family of value functions whose envelope is the market hedonic or implicit price function.

The model is easily expanded to include several quantities, so long as consumers are restricted to purchasing only one model. Following Houthakker (1952), the utility function becomes $U\left(x_{1}, z_{1}, \ldots, z_{n}, m\right)$, where $m$ is the number of units consumed of a model with characteristics $z$. The constraint is $y=x+m p(z)$, and necessary conditions become

$$
\begin{gathered}
\frac{\partial U}{\partial m}=-p(z) U_{x}+U_{m}=0, \\
\frac{\partial U}{\partial z_{i}}=-m p_{i}(z) U_{x}+U_{z_{i}}=0 .
\end{gathered}
$$

The value function is still defined as the amount a consumer is willing to pay for $z$ at a fixed utility index but now with the proviso that $m$ is optimally chosen. That is, $\theta\left(z_{1}, \ldots, z_{n}\right)$ is defined by eliminating $m$ from

$$
\begin{gathered}
u=U\left(y-m \theta, z_{1}, \ldots, z_{n}, m\right) \\
U_{m} / U_{\boldsymbol{x}}=\theta .
\end{gathered}
$$

Again, $\theta_{z_{i}}$ is proportional to $U_{z_{i}} / U_{x}$. The logic underlying figure 1 remains intact, and it can just as well serve for this case. However, second-order 
conditions are now more complex. For example, convexity of $p(z)$ is no longer sufficient for a maximum as it was in the case where $m$ was restricted to be unity. Also, it is necessary to employ stronger assumptions than those used above if the value function $\theta$ is to be concave.

Note there is no question of monopsony involved here. Consumers act competitively in spite of the fact that marginal cost of quality, $p_{i}(z)$, is not necessarily constant-it is increasing in figure 1-because as many units as desired of any brand can be purchased without affecting prices. The function $p(z)$ is the same for all buyers and independent of $m$.

\section{B. The Production Decision}

Having set up the formal apparatus above, we give a symmetrical and consequently brief account of producers' locational decisions. What package of characteristics is to be assembled? Let $M(z)$ denote the number of units produced by a firm of designs offering specification $z$. The discussion is limited to the case of nonjoint production, in which each production establishment within the firm specializes in one design, and there are no cost spillovers from plant to plant. Thus a "firm" is an arbitrary collection of atomistic production establishments, each one acting independently of the others. Analytical difficulties arising from true joint production are noted in passing.

Total costs in an establishment are $C(M, z ; \beta)$, derived from minimizing factor costs subject to a joint production function constraint relating $M, z$, and factors of production. The shift parameter $\beta$ reflects underlying variables in the cost minimization problem, namely, factor prices and production function parameters. Assume $C$ is convex with $C(0, z)=0$ and $C_{M}$ and $C_{z_{i}}>0$. There are no production indivisibilities, and marginal costs of producing more units of a model of given design are positive and increasing. Similarly, marginal costs of increasing each component of the design are also positive and nondecreasing. (Ordinarily, there will be some technological constraints that limit the set of feasible locations on the plane.) Each plant maximizes profit $\pi=M p(z)-$ $C\left(M, z_{1}, \ldots, z_{n}\right)$ by choosing $M$ and $z$ optimally, where unit revenue on design $z$ is given by the implicit price function for characteristics, $p(z) .^{4}$

\footnotetext{
${ }^{4}$ Our inability to treat joint production nontrivially yet simply stems from the spectrum-of-commodities assumption. If a finite number (say $v$ ) of packages is available, it would be straightforward formally to specify a standard $v$-component multiple product cost function for the firm, and proceed on that basis. In the present case, firms engage in joint production only insofar as they own establishments specializing in different packages. However, genuine joint production requires cost dependencies between production units within the firm: the firm must choose a function $M(z)$ describing an entire "product line" offered in the market. The entire function $M(z)$ is an argument in each plant's costs and total costs in turn are the sum (or integral) over all production establishment costs. A complete treatment requires use of functional analysis and is beyond the scope of this paper.
} 
Again, firms are competitors and not monopolists even though marginal costs of attributes $p_{i}(z)$ are not necessarily constant because all establishments observe the same prices and cannot affect them by their individual production decisions: $p(z)$ is independent of $M$.

Optimal choice of $M$ and $z$ requires

$$
\begin{gathered}
p_{i}(z)=C_{z_{i}}\left(M, z_{1}, \ldots, z_{n}\right) / M, \quad i=1, \ldots, n \\
p(z)=C_{M}\left(M, z_{1}, \ldots, z_{n}\right) .
\end{gathered}
$$

At the optimum design, marginal revenue from additional attributes equals their marginal cost of production per unit sold. Furthermore, quantities are produced up to the point where unit revenue $p(z)$ equals marginal production cost, evaluated at the optimum bundle of characteristics. As above, convexity of $C$ does not assure second-order conditions due to nonlinearity of $p(z)$, and some stronger conditions, assumed to be satisfied in what follows, are required (see Intriligator 1971).

Symmetrically with the treatment of demand, define an offer function $\phi\left(z_{1}, \ldots, z_{n} ; \pi, \beta\right)$ indicating unit prices (per model) the firm is willing to accept on various designs at constant profit when quantities produced of each model are optimally chosen. A family of production "indifference" surfaces is defined by $\phi$. Then $\phi\left(z_{1}, \ldots, z_{n} ; \pi, \beta\right)$ is found by eliminating $M$ from

$$
\pi=M \phi-C\left(M, z_{1}, \ldots, z_{n}\right)
$$

and

$$
C_{M}\left(M, z_{1}, \ldots, z_{n}\right)=\phi
$$

and solving for $\phi$ in terms of $z, \pi$, and $\beta$. Differentiate (8) and (9) to obtain $\phi_{z_{i}}=C_{z_{i}} / M>0$ and $\phi_{\pi}=1 / M>0$.

The marginal reservation supply price for attribute $i$ at constant profit, assumed increasing in $z_{i}$, is $\phi_{z_{i}}$. Again convexity of $C$ does not always guarantee $\phi_{z_{i} z_{i}}>0$. Since $\phi$ is the offer price the seller is willing to accept on design $z$ at profit level $\pi$, while $p(z)$ is the maximum price obtainable for those models in the market, profit is maximized by an equivalent maximization of the offer price subject to the constraint $p=\phi$. Thus maximum profit and optimum design satisfy $p_{i}\left(z^{*}\right)=$ $\phi_{z_{i}}\left(z_{1}^{*}, \ldots, z_{n}^{*} ; \pi^{*}, \beta\right)$, for $i=1, \ldots, n$, and $p\left(z^{*}\right)=\phi\left(z_{1}^{*}, \ldots, z_{n}^{*}\right.$; $\left.\pi^{*}, \beta\right)$. Producer equilibrium is characterized by tangency between a profit-characteristics indifference surface and the market characteristicsimplicit price surface.

One dimension of the solution is depicted in figure 2, where

$$
\phi\left(z_{1}, z_{2}^{*}, \ldots, z_{n}^{*} ; \pi, \beta\right)
$$




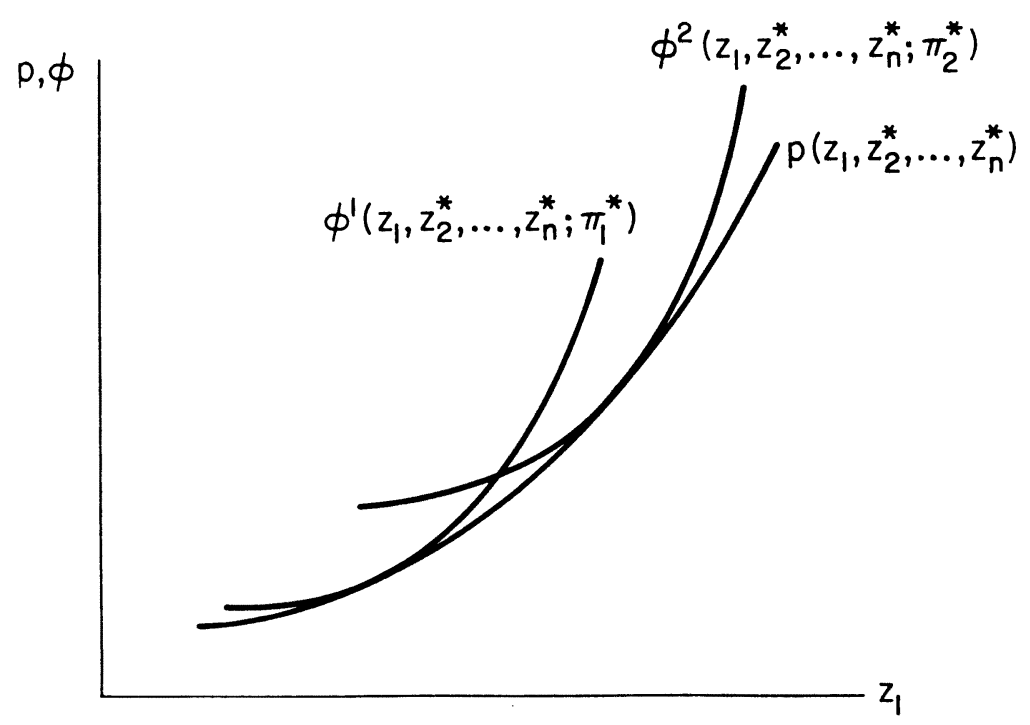

FIG. 2

defines a family of curves on the $z_{1}-\phi$ plane cut through the indifference surface at the optimum values of the other attributes. Only one member is shown in the figure. The curve labeled $\phi^{1}$ refers to a production unit possessing production and cost conditions making it well suited to produce lesser amounts of $z_{1}$, while the one labeled $\phi^{2}$ refers to a firm with a comparative advantage at producing higher values of $z_{1}$. That is, the two plants have distinct values of the parameter $\beta$. More generally, there is a distribution of $\beta$ across all potential sellers. Let $G(\beta)$ represent that distribution. Then producer equilibrium is characterized by a family of offer functions that envelop the market hedonic price functions.

What is the empirical content of $\beta$ ? It is anything that shifts cost conditions among firms. Thus, differences in factor prices are one possibility. For example, many products are produced in several countries and are traded on national markets (for examples, see Griliches [1971], chap. 5). There is no reason to assume equalization of factor prices in these cases. More generally, anything allowing identification of conventional multiproduct production functions in cross-section data serves to provoke differences in $\beta$. Factor price differences across states or regions within a country often serve this purpose and do so here as well. Second, differences in "technology," as reflected by typically unmeasured, firm-specific factors of production, also act as supply shifters across firms. For example, agricultural production function research often treats education of the farm operator in this manner. Firm-specific R\&D expenditure as well as the phenomena of progress-function-learning also serve these purposes. 


\section{What Do Hedonic Prices Mean?}

An answer to the question is an immediate application of the above analysis. Superimpose figure 2 onto figure 1. In equilibrium, a buyer and seller are perfectly matched when their respective value and offer functions "kiss" each other, with the common gradient at that point given by the gradient of the market clearing implicit price function $p(z)$. Therefore, observations $p(z)$ represent a joint envelope of a family of value functions and another family of offer functions. An envelope function by itself reveals nothing about the underlying members that generate it; and they in turn constitute the generating structure of the observations. Some qualifications are necessary however. (a) Suppose there is no variance in $\beta$ and all firms are identical. Then the family of offer functions degenerates to a single surface, and $p(z)$ must be everywhere identical with a unique offer function. Price differences between various packages are exactly equalizing among sellers because offer functions are constructed at constant profit. A variety of packages appear on products markets to satisfy differences in preferences among consumers, and the situation persists because no firm finds it advantageous to alter the quality content of its products. (b) Suppose sellers differ, but buyers are identical. Then the family of value functions collapses to a single function and is identical with the hedonic price function. Observed price differences are exactly equalizing across buyers, and $p(z)$ identifies the structure of demand.

\section{Existence of Market Equilibrium}

Analysis of consumer and producer decisions has proceeded on the assumption of market equilibrium. This section demonstrates some details of equilibrium price and quantity determination. Market quantity demanded for products with characteristics $z$ is $Q^{d}(z)$, and $Q^{s}(z)$ is market quantity supplied with those attributes. It is necessary to find a function $p(z)$ such that $Q^{d}(z)=Q^{s}(z)$ for all $z$, when buyers and sellers act in the manner described above. The fundamental difficulty posed by this problem is that $Q^{d}(z)$ and $Q^{s}(z)$ depend on the entire function $p(z)$. For example, suppose quantities demanded and supplied at a particular location do not match at prevailing prices. The effect of a change in price at that point is not confined to models with those particular characteristics but induces substitutions and locational changes everywhere on the plane. A very general treatment of the problem is found in Court (1941), and our discussion is devoted to some examples. These examples have been chosen for their simplicity but illuminate the problem and illustrate most of the basic issues. In contrast to the rest of the paper, discussion is specialized to the case where goods are described by exactly one attribute (i.e., $n=1$ ). Therefore $z_{1}$ represents an unambiguous 
measure of "quality." When $n=1$, the location surface degenerates to a line rather than a plane, and products are unequivocally ranked by their $z$ content.

\section{A. Short-Run Equilibrium}

Consider a short-run equilibrium in which firms have geared up for the quality $\left(z_{1}\right)$ of goods they can produce and are only capable of varying quantities. The horizon is sufficiently short so that new entry is precluded, and the distribution of firms by quality is given as an initial condition. The market reveals an implicit price function $p\left(z_{1}\right)$, and each firm determines the quantity it supplies to the market according to condition (7). Market supply in a small interval $d z_{1}$ near quality $z_{1}$ is found by weighting firm supply by the quality distribution function. Consumers differ in tastes and income, but all determine optimal quality and quantity as in (4) and (5). Market demand near any quality $z_{1}$ is found by using the conditions of consumer equilibrium to transform the distribution of tastes and income into a distribution of qualities demanded and weighting individual quantities demanded by the resulting distribution of qualities. Finally, setting demand equal to supply yields a differential equation in $p$ and $z_{1}$ that must be satisfied by market equilibrium, subject to some boundary conditions.

To be specific, assume that $C(N, z)=(a / 2) M^{2} z_{1}^{2}$ for all firms. Also, suppose firms are uniformly distributed by the characteristic $z_{1}$ : $g\left(z_{1}\right) d z_{1}=k d z_{1}$ for $z_{1 s} \leq z_{1} \leq z_{1 l}$, where $k$ is a constant and $z_{1 l}$ and $z_{1 s}$ are exogenously determined upper and lower limits of the product line. Apply equation (7) to obtain firm supply: $M\left(z_{1}\right)=p / a z_{1}^{2}$, since qualities cannot be varied by assumption. Therefore,

$$
Q^{s}\left(z_{1}\right) d z_{1}=g\left(z_{1}\right) M\left(z_{1}\right) d z_{1}=\left[(k / a) p\left(z_{1}\right) / z_{1}^{2}\right] d z_{1} .
$$

Assume a fixed number of consumers in the population and that only one unit per customer of the optimal model is purchased. Consumers have the same income, and utility is linear in $x$ and $z_{1}$, with the marginal rate of substitution, $\rho$, varying from person to person. Maximize $U\left(x, z_{1}\right)=$ $x+\rho z_{1}$ subject to $y=x+p\left(z_{1}\right)$. Each consumer purchases a brand for which $d p / d z_{1}=p^{\prime}\left(z_{1}\right)=\rho$. In this case the value functions of figure 1 are straight lines with a different slope, $\rho$, for each person. The marginal condition characterizes consumer choice so long as $p^{\prime \prime}>0$, which will be shown to be true. Suppose $\rho$ is distributed uniformly, $f(\rho) d \rho=b d \rho$ for $\rho_{s} \leq \rho \leq \rho_{l}$, where $b$ is a constant and $\rho_{l}$ and $\rho_{s}$ are, respectively, the largest and smallest marginal rates of substitution in the population. Use the marginal condition $p^{\prime}=\rho$ to transform $f(\rho) d \rho$ into 


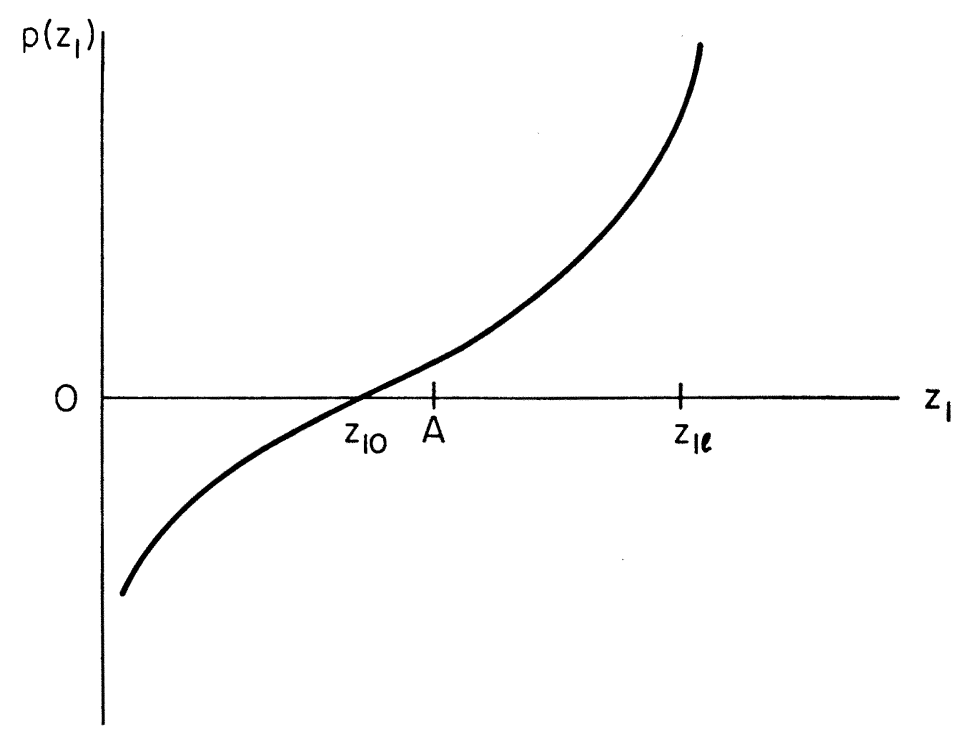

Fig. 3

a distribution of $z_{1}$. Then

$$
Q^{d}(z) d z=f\left(z_{1}\right)\left|\frac{d p}{d z_{1}}\right| d z_{1}=b p^{\prime \prime}\left(z_{1}\right) d z_{1} .
$$

Price must clear the market at every quality. Equating (10) and (11), $p\left(z_{1}\right)$ must satisfy the differential equation

$$
(k \mid b a) p / z_{1}^{2}=d^{2} p / d z_{1}^{2} .
$$

Equation (12) is a special case of what is called "Euler's equation" and has a known solution of the form

$$
p=c_{1} z_{1}^{r}+c_{2} z_{1}^{s},
$$

where $c_{1}$ and $c_{2}$ are constants determined by the boundary conditions and $r$ and $s$ are defined by $r^{2}-r-(a / b k)=0: r=(1+\sqrt{1+4 a / b k}) / 2$ and $s=(1-\sqrt{1+4 a / b k}) / 2$. The parameters $r$ and $s$ are real numbers and $r>0$ and $s<0$. Furthermore, $p^{\prime}\left(z_{1}\right)$ would not be positive throughout its range unless $c_{1}>0$ and $c_{2}<0$, and consumers ${ }^{\cdot}$ could not be interior at those points. Equation (13) is graphed in figure 3 on that assumption. Note that $p$ in (13) exhibits an inflection point at $z_{10}=$ $\left(-c_{1} / c_{2}\right)^{1 /(r-s)}$, and it so happens that $p\left(z_{10}\right) \equiv 0$. Therefore $p^{\prime \prime}>0$ for $z_{1}>z_{10}$.

Boundary conditions.-Competition requires there be no masses of consumers at any quality, for there are few sellers located at any point and 
they would otherwise add nonzero weight to the market. As seen in Section II, consumers with high values of $\rho$ buy higher-quality models, and it must be true that those for whom $\rho=\rho_{l}$ purchase the highest quality available. Otherwise prices of quality $z_{1 l}$ would fall, a great mass of consumers would switch over to them, driving the price back up and causing those buyers to relocate again. Therefore, one boundary condition is $p^{\prime}\left(z_{1 l}\right)=\rho_{l}$, or

$$
\rho_{l}=r c_{1} z_{1 l}^{r-1}+s c_{2} z_{1 l}^{s-1} .
$$

The other boundary condition is found by examining the lower end of the line. The following three cases cover all relevant possibilities:

1. $z_{1 s}=0$ and $\rho_{s}>0$. Firms choose not to sell at negative prices (see fig. 3) and all plants geared to produce qualities less than $z_{10}$ (to be determined) shut down. On the other hand, all consumers value $z_{1}$ at least as much as its minimum supply price (i.e., zero) and it must be true that they all buy some value of $z_{1}$. Individuals for whom $\rho=\rho_{s}$ consume the lowest qualities appearing on the market, for if they chose qualities greater than $z_{10}$, prices of models in the neighborhood of $z_{10}$ would fall to zero, inducing low $\rho$ customers to relocate there and driving their prices back up. Thus a second boundary condition is $p^{\prime}\left(z_{10}\right)=\rho_{s}$, or

$$
\rho_{s}=r c_{1} z_{10}^{r-1}+s c_{2} z_{10}^{s-1} \text {. }
$$

The parameters $z_{10}, c_{1}$, and $c_{2}$ are determined by equations (14) and (15) plus the definition of $z_{10}$. It can be shown that $c_{1}>0$ and $c_{2}<0$, as required by the second-order conditions of consumer equilibrium. Therefore, the equilibrium hedonic price function appears as a portion of the curve in figure 3 in the interval $\left(z_{10}, z_{1 l}\right)$.

2. If $\rho_{s}=0=z_{1 s}$, all producers must be in the market, and it follows that $z_{10}=0$. This only is possible if $p^{\prime}(0)=\rho_{s}=0$ and $c_{2}$ must be zero. In this case price is a log-linear function of quality.

3. $z_{1 s}>0$ and $\rho_{s}=0$. Now some consumers do not value $z_{1}$ very highly, and there is a definite limit to the smallest amount available. Clearly, $p\left(z_{1 s}\right)$ must exceed zero and some consumers must be driven out of the market, finding it optimal not to consume the product at all. If not, consumers with small values of $\rho$ would mass on $z_{1 s}$ (there would be a corner solution there), adding finite weight to the market and causing $p\left(z_{1 s}\right)$ to explode. Using the budget constraint, the market rate of exchange between not buying at all and buying $z_{1 s}$ is $\left[y-p\left(z_{1 s}\right)\right] / z_{1 s}$ and must equal the slope of the value function for buyers at that (extensive) margin. That is, the condition $\left[y-p\left(z_{1 s}\right)\right] / z_{1 s}=p^{\prime}\left(z_{1 s}\right)$ replaces equation (15) after substituting for $p$ and $p^{\prime}$ from (13) -in the determination of $c_{1}$ and $c_{2}$. The hedonic price function also can be illustrated in figure 3 as the portion of the curve between the points such as those marked $A\left(=z_{1 s}\right)$ 
and $z_{1 l}$. Again, $c_{1}$ and $c_{2}$ have the correct signs and the second-order conditions are fulfilled.

A second type of short-run equilibrium could be considered in which existing firms can alter qualities as well as quantities of their products. When there is a distribution of cost functions, it is necessary to proceed analogously to the treatment of demand in the example above. For example, costs might be described by $(a / 2) N^{2} z_{1}^{\lambda}$ with $\lambda$ varying across firms. Then $(\lambda / 2)=z_{1} p^{\prime} \mid p$ is used to transform the distribution of $\lambda$ into a distribution of qualities supplied. The resulting distribution weights firm quantities supplied in the determination of market supply at any quality. A little experimentation will show that the differential equation resulting from setting $Q^{d}\left(z_{1}\right)=Q^{s}\left(z_{1}\right)$ is nonlinear in most cases, and closed solutions are not always feasible.

\section{B. Long-Run Equilibrium}

Firms may vary qualities at will and also construct establishments of optimum size. No entry restrictions imply the absence of profit $\left(\pi^{*}=0\right)$ and long-run offer price for each firm must satisfy $\phi(z ; \beta)=$ $C(M, z ; \beta) / M$. Plants are constructed to produce models of quality $z$ at minimum cost. Hence scale economies are exhausted under competition and the optimum production unit occurs where $C(M, z, \beta)$ is linear in $M$, variations of quantity being achieved by changes in the number of establishments. Let $h(z ; \beta)$ represent minimum average cost of $z$ for an establishment of optimum size. Then $C(M, z ; \beta)=M h(z ; \beta)$ in the long run. Therefore $\phi=h(z ; \beta)$ and $p(z)=h(z ; \beta)$ is the equilibrium condition for maximum profit and $p(z)$ is completely determined by supply, or by the envelope of the family $h(z ; \beta)$ with respect to $\beta$. Generalization to $n$ characteristics is obvious in this case.

\section{An Identification Problem}

Section III demonstrated that complete solutions for $p(z)$ and the distribution of qualities traded sometimes can be obtained if sufficient a priori structure is imposed on the problem. However, it is not always possible to proceed in that manner. In general, the differential equation defining $p(z)$ is nonlinear and it may not be possible to find closed solutions. Moreover, a great deal of structure must be imposed. For example, the distribution of income follows no simple law throughout its range, making it difficult to specify the problem completely. Finally, partialdifferential equations must be solved when there is more than one characteristic. This section sketches an alternative and more efficient procedure, based on the analysis of Section II. 


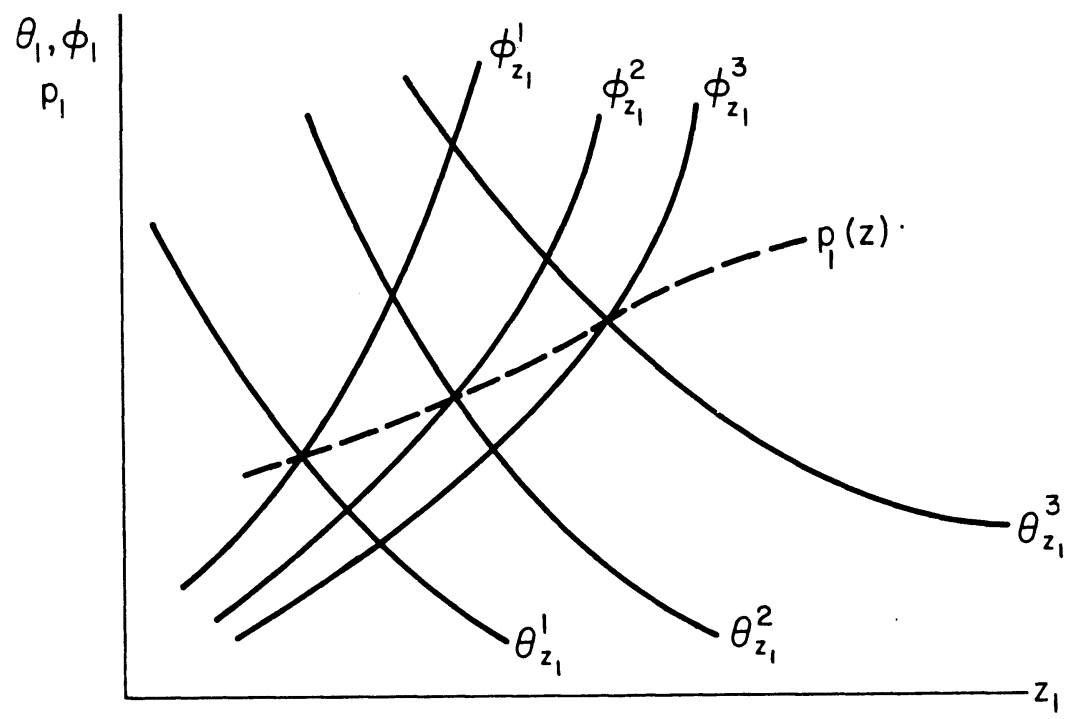

Fig. 4

As shown above, derivatives of a consumer's value function, $\theta_{z_{i}}$, are proportional to marginal rates of substitution. They are reservationdemand prices for additional amounts of $z_{i}$ at a constant utility index. Therefore $\left\{\theta_{z_{i}}(z)\right\}$ are the inverses of a set of ordinary compensated demand functions for the $z_{i}$ 's. The marginal cost of $z_{i}$ to the consumer is $p_{i}(z)$, and optimal $z$ is determined where marginal costs equal marginal values. One dimension of these marginal concepts is illustrated in figure 4. The curves labeled $\theta_{z_{i}}^{j}$ are derivatives of $\theta^{j}$ in figure 1 and reflect compensated demand functions for various buyers. The dashed line labeled $p_{1}(z)$ is the common marginal cost confronting all buyers. Consumer choice is given by the intersection of demand and marginal cost. It should be emphasized that the functions $\theta_{z_{i}}(z)$ are compensated demand prices (real income held constant) and can only be derived once equilibrium is determined, as in Section II. For example, a new equilibrium resulting from an exogenous shift in $p$ would not always be given by the intersection of the new marginal costs, $p_{1}(z)$, and the initial compensated demand price functions. An exception occurs when $\theta_{z_{i} u}=0$ and the family of surfaces $\theta(z ; u)$, such as depicted in figure 1 , are all parallel to each other: $\theta_{z_{i} u}=0$ is equivalent to constant marginal utility of money and $\theta_{z_{i}}$ is unique and independent of $u$ only in that case. If $\theta_{z_{i} u} \neq 0$, the shape and location of the $\theta_{z_{i}}^{j}$ functions are determined by the equilibrium conditions of Section II : tangency between $p(z)$ and $\theta^{j}\left(z, u^{*}\right)$.

A similar procedure applies to firms: $\theta_{z_{i}}$ is the reservation supply price of incremental $z_{i}$ and reflects a profit-compensated supply function for 
characteristic $z_{i} ; p_{i}$ is the marginal revenue function for $z_{i}$ facing each firm. One dimension of producer equilibrium is shown in figure 4 as the intersection of a set of compensated supply curves for various firms, $\theta_{z}^{j}$, with a common marginal revenue function, $p_{1}(z)$.

Figure 4 reiterates the major conclusion of Section II in terms of derivatives of $p(z)$. Equlibrium is described by the intersection of supply and demand functions. However, income effects have been removed, in distinction to the typical case. Observed marginal hedonic prices merely connect equilibrium reservation prices and characteristics and reveal little about underlying supply and demand functions.

However, figure 4 suggests a method that can be used for estimation. In principle, data are available on designs purchased by buyers and also on their incomes and taste variables such as age, education, etc. Denote these empirical counterparts of $\alpha$ by a vector $Y_{1}$. Data are also potentially available on the characteristics' content of models produced by sellers and factor price and specific technological differences among them. Denote the empirical counterparts of $\beta$ by a vector $Y_{2}$. Following figure 4, let $F_{i}\left(z, Y_{1}\right)$ represent the marginal demand price for $z_{i}$ and $G_{i}\left(z, Y_{2}\right)$ represent the marginal supply price. Ignoring random terms, the model to be estimated can be written as

$$
\begin{array}{ll}
p_{i}(z)=F^{i}\left(z_{1}, \ldots, z_{n}, Y_{1}\right) & \text { (demand), } \\
p_{i}(z)=G^{i}\left(z_{1}, \ldots, z_{n}, Y_{2}\right) & \text { (supply), }
\end{array}
$$

for $i=1, \ldots, n$, where $p_{i}$ and $z_{i}$ are all jointly dependent variables and $Y_{1}$ and $Y_{2}$ are exogenous demand and supply shift variables. The $2 n$ equations determine the $2 n$ endogenous variables $p_{i}$ and $z_{i}$. Estimation requires a two-step procedure. First, estimate $p(z)$ by the usual hedonic method, without regard to $Y_{1}$ and $Y_{2}$. That is, regress observed differentiated products' prices, $p$, on all of their characteristics, $z$, using the best fitting functional form. This econometrically duplicates the information acquired by agents in the market, on the basis of which they make their decisions. Denote the resulting estimate of the function $p(z)$ by $\hat{p}(z)$. Next, compute a set of implicit marginal prices, $\partial p(z) / \partial z_{i}=\hat{p}_{i}(z)$ for each buyer and seller, evaluated at the amounts of characteristics (numerical values of $z$ ) actually bought or sold, as the case may be. Finally, use estimated marginal prices $\hat{p}_{i}(z)$ as endogenous variables in the secondstage simultaneous estimation of equations (16) and (17). Estimation of marginal prices plays the same role here as do direct observations on prices in the standard theory and converts the second-stage estimation into a garden variety identification problem. There are four cases to consider:

1. There is no variance in $\beta$ and cost conditions are identical across firms. The variables $Y_{2}$ drop out of equation (17) and $\hat{p}(z)$ identifies the 
offer function. Similarly, the sample observations on $\hat{p}_{i}(z)$ and the $z_{i}$ identify compensated supply functions. Suppose several cross sections for different years are available and firms' production functions have been subject to technical change. Then within-year hedonic price regressions identify supply conditions for each year. Changes in marginal prices and qualities induced by changing technology and cost conditions between years approximately sweep out the structure of preferences and compensated demand functions (with due qualification for the nonconstancy of the marginal utility of money).

2. If buyers are identical, but sellers differ, $Y_{1}$ drops out of (16) and single cross-sectional observations trace out compensated demand functions.

3. If buyers are identical and so are sellers, offer and value functions are tangent at a single point, and only one quality appears on the market. The observations degenerate to a single point; there is no product differentiation and no problem.

4. In general there is both a distribution of buyers and another distribution of sellers. Both $Y_{1}$ and $Y_{2}$ have nonzero variance, and the usual identifying rank and order conditions apply. A necessary prior condition for estimation is that $\hat{p}(z)$ be nonlinear at stage one. For if $\hat{p}(z)$ happens to be linear, $\hat{p}_{i}(z)$ are constants, independent of qualities traded, and display zero variance across sample observations. As shown above, linearity of $p(z)$ is unlikely so long as there is increasing marginal cost of attributes for sellers and it is not possible to untie packages. But it is obvious that the model does not apply if very few distinct products are actually traded.

\section{Price Indexes, Economic Welfare, and Legislated Restrictions}

This section uses the model to analyze the welfare consequences of qualitystandards legislation, a problem not easily handled by conventional methods. The discussion clarifies issues in recent controversies regarding treatment of legislated standards in the construction of price indexes. For example, how should mandatory installation of seat belts and air bags affect the automobile price index? For expository convenience, discussion is confined to the case of one attribute. Generalization to several characteristics is immediate.

A minimum quality standard means that $z \geq \bar{z}$, and brands containing less than $\bar{z}$ are prohibited from the market. Assume constant returns to quantities (as in Section III B). Then the law is irrelevant for all consumers previously purchasing packages containing more than the legislated minimum. The situation for a buyer whose choice is affected by the law is shown in figure $5: z^{*}$ was the original choice, whereas $\bar{z}$ is chosen after the law has been passed, since $z^{*}$ is no longer available. The 


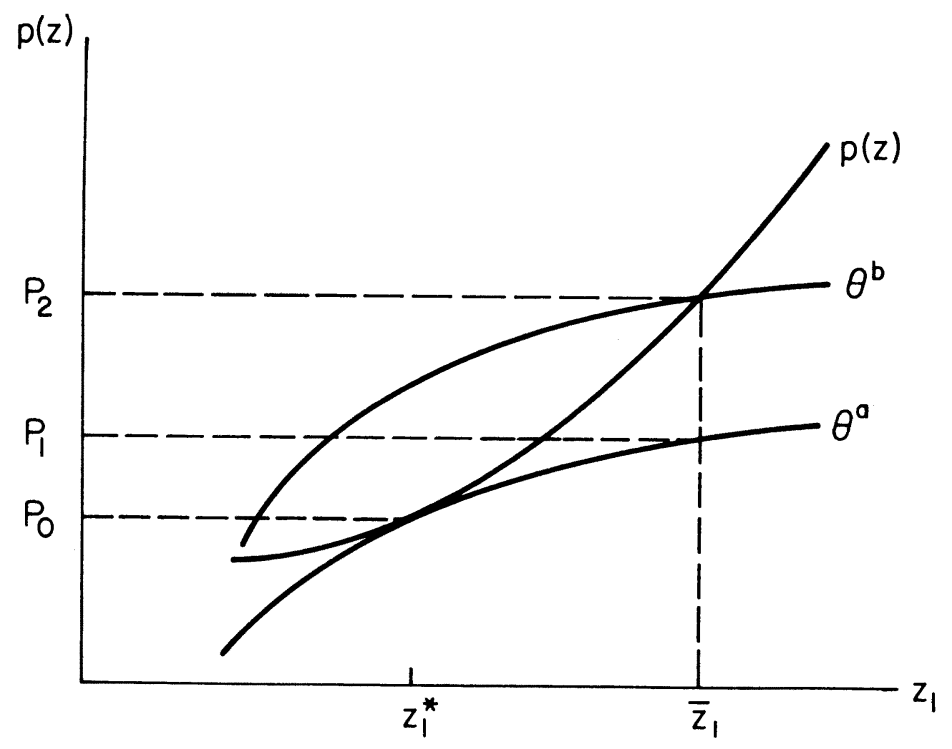

FIG. 5

minimum attainable value function has shifted from $\theta^{a}$ to $\theta^{b}$, and the consumer is worse off (see eq. [2]).

Choose the distance $\Delta P=P_{2}-P_{1}$ as a monetary measure of the loss in welfare. Since $\partial \theta / \partial y=1, \Delta P$ is the bribe necessary for the consumer to purchase $\bar{z}$ when $z^{*}$ was available. Clearly, this measure is not unique (i.e., if compensation is evaluated at a different amount of $z$ ) unless $\theta_{z_{i} u}=0$. The welfare loss can be estimated from the implicit price and bid functions. The distance $P_{2}-P_{0}$ is given by

$$
\int_{z_{1}^{*}}^{z_{1}} p_{1}(z) d z
$$

or the area under marginal cost from $z^{*}$ to $\bar{z}$, and is shown in figure 6 as $z_{1}^{*} a b \bar{z}_{1}$. It represents the social opportunity cost of additional resources necessary to produce $\bar{z}$ instead of $z^{*}$. The integral

$$
\int_{z^{*}}^{z} \theta_{1}(z) d z
$$

or the area under a compensated demand function (compensated at the original level of real income) between $z^{*}$ and $\bar{z}$ in figure $6\left(z_{1}^{*} a c \bar{z}_{1}\right)$ measures the amount the consumer would have paid for the increment $\left(\bar{z}_{1}-z_{1}^{*}\right)$ at the unrestricted level of welfare. It measures $P_{1}-P_{0}$ in figure 5 and represents the benefit of the restriction. The difference 


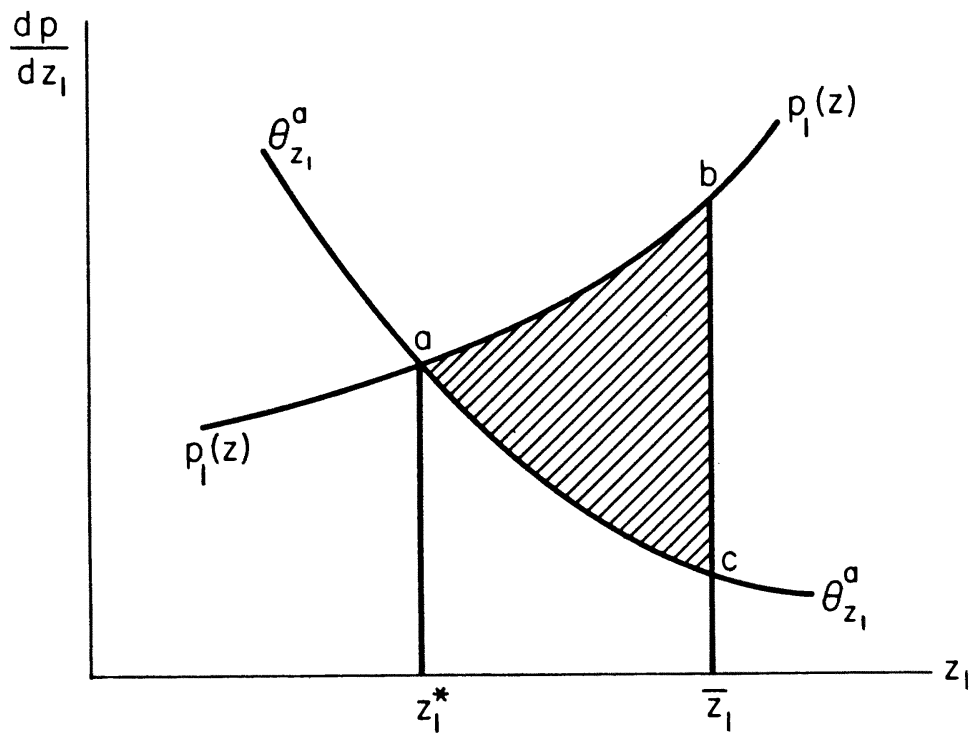

Fig. 6

between costs and benefits is given by $P_{2}-P_{1}$, or the difference between the areas under the marginal cost and compensated demand functions, the shaded area in figure 6 . In the general case of several attributes, $\Delta P$ must be measured by a line integral. Otherwise, everything else is unchanged.

When the marginal utility of money is constant, $\Delta P$ is unique and the price restriction is equivalent to an additive increase in implicit prices in amount $\Delta P$ everywhere. In figure $5, \theta_{z u}=0$ means that all value functions are parallel, and if the budget constraint was $y=x+p(z)+\Delta P$ instead of $y=x+p(z)$, the consumer would have arrived exactly at $\theta^{b}$ of his own free choice. The real price of the characteristic has risen because choices are restricted, and the price index should rise to reflect that fact. A natural measure of the real price increase imposed by the law is a weighted average of terms such as $\Delta P$ (including buyers for whom $\Delta P=$ $0)$, where the weights are expenditure shares among all consumers. ${ }^{5}$ This measure overstates the loss insofar as the restriction actually forces

${ }^{5} \mathrm{~A}$ complete assessment of the law and its effect on the price index requires balancing the costs calculated above against any externality-induced social benefits of the restriction. In our judgment, seat belts and air bags are in a different category than emissioncontrol devices. In regard to the latter, the apparatus above can be used easily to analyze the effect of the European system of taxing engine displacement. An ad valorem tax increases average and marginal costs of packages with larger liter capacity, and the usual income and substitution effects apply: packages with smaller amounts of this and complementary characteristics (such as size of car) are purchased. 
some consumers completely out of the generic goods market since they escape the full loss $\Delta P$. Also, standard index number problems arise when the marginal utility of money is not constant.

\section{Conclusions}

This paper has drawn out the observational consequences of the construct of implicit markets for characteristics embodied in differentiated products. When goods can be treated as tied packages of characteristics, observed market prices are also comparable on those terms. The economic content of the relationship between observed prices and observed characteristics becomes evident once price differences among goods are recognized as equalizing differences for the alternative packages they embody. Here, as elsewhere, price differences generally are equalizing only on the margin and not on the average. Hence, estimated hedonic price-characteristics functions typically identify neither demand nor supply. In fact, those observations are described by a joint-envelope function and cannot by themselves identify the structure of consumer preferences and producer technologies that generate them.

The formal analysis is complicated by the fact that budget constraints are nonlinear. Consequently, it is not surprising that far weaker theorems than usual apply. However, a feasible econometric procedure for estimating the underlying generating structure has been derived through the use of derivative transformations. When constraints are nonlinear, marginal prices serve the same role as average prices do in the linear case. Finally, the essential spatial context of the problem means that substitution and income effects must be more carefully distinguished than usual. Indeed, here is a major practical instance where compensated demand and supply functions become the relevant fundamental concepts. These compensated functions are estimated by the econometric method and measures of consumer and producer surplus can be derived directly from them. We anticipate that the basic conceptual framework outlined above will have a variety of applications to many practical problems involving equilibrium in cross-section data.

The analysis has been simplified by assuming divisibility in production. Generalization has to incorporate nonconvexities, and discontinuities must result. When nonconvexities are not small relative to the market, it is obvious that only isolated locations on the characteristics surface will be filled. In other words, such a generalization will naturally incorporate the case of monopolistic competition, and observed "distances" (in terms of characteristics) between differentiated products will be endogenously determined. The methods employed above do not carry through because certain nonmarginal decisions must be analyzed, and far more sophisticated techniques are required. 


\section{References}

Alonzo, William. Location and Land Use. Cambridge, Mass.: Harvard Univ. Press, 1964.

Becker, Gary S. "A Theory of the Allocation of Time." Econ. J. 75 (September 1965) : 493-517.

Court, Louis M. "Entrepreneurial and Consumer Demand Theories for Commodity Spectra." Econometrica 9, no. 1 (April 1941): 135-62; no. 2 (JulyOctober 1941): 241-97.

Ellickson, Bryan. "Jurisdictional Fragmentation and Residential Choice." A.E.R. 61 (May 1971): 334-39.

Gordon, Robert J. “The Measurement of Durable Goods Prices." Mimeographed. Nat. Bur. Econ. Res., 1973.

Griliches, Zvi, ed. Price Indexes and Quality Change. Cambridge, Mass.: Harvard Univ. Press, 1971.

Houthakker, H. S. "Compensated Changes in Quantities and Qualities Consumed." Rev. Econ. Studies 19, no. 3 (1952): 155-64.

Intriligator, Michael D. Mathematical Optimization and Economic Theory. Englewood Cliffs, N.J.: Prentice-Hall, 1971.

Lancaster, Kelvin J. "A New Approach to Consumer Theory." J.P.E. 74 (April 1966) : 132-56.

Lewis, H. Gregg. "Interes del empleador en las horas de Trabajo del empleado" [Employer interests in employee hours of work]. Cuadernos de Economia, Catholic Univ. Chile, 1969.

Lipsey, Richard G., and Rosenbluth, Gideon. "A Contribution to the New Demand Theory: A Rehabilitation of the Giffen Good." Canadian J. Econ. 4 (May 1971): 131-63.

Muth, Richard F. "Household Production and Consumer Demand Functions." Econometrica 34 (July 1966) : 699-708.

Ohta, Makoto, and Griliches, Zvi. "Makes and Depreciation in the U.S. Passenger Car Market." Mimeographed. Harvard Univ., 1972.

Tiebout, Charles M. "A Pure Theory of Local Expenditure." J.P.E. 64 (October 1956) : 416-24. 